\title{
Choroid Plexus Carcinoma with Rhabdoid Features: A Case Report
}

\author{
Yohana Mateo-Camacho ${ }^{1}$, Ramón Figueroa-Ortiz ${ }^{2}$, Julita Orozco-Vázquez ${ }^{1}$, Angélica \\ Moreno-Blanco ${ }^{1}$, and Aureliano Plácido-Mendez ${ }^{1}$ \\ ${ }^{1}$ Centro Medico Nacional 20 de Noviembre \\ ${ }^{2}$ Augusta University Medical College of Georgia
}

October 3, 2021

\begin{abstract}
The case of a patient under 2 years of age with acute vomiting, fever and seizures. MR imaging of the brain revealed a right lateral intraventricular mass and mild hydrocephalus. Surgery achieved gross total tumor resection, but tumor histology revealed choroid plexus carcinoma with heavy stratification and atypical "rhabdoid" cells.
\end{abstract}

\section{Choroid Plexus Carcinoma with Rhabdoid Features: A Case Report SUMMARY}

We present the case of a patient under 2 years of age with acute vomiting, fever and seizures. MR imaging of the brain revealed a right lateral intraventricular mass and mild hydrocephalus. Surgery achieved gross total tumor resection, but tumor histology revealed choroid plexus carcinoma with heavy stratification and atypical "rhabdoid" cells. Choroid plexus carcinomas are extremely rare tumors, and the presence of rhabdoid cells is even rarer. Correct identification of choroid plexus carcinomas is essential since the management and prognosis are very different from benign choroid plexus tumors. Although they seem morphologically similar, choroid plexus papilloma and choroid plexus carcinoma have subtle differences that, once identified, allow an appropriate diagnosis. We present also a review of current literature highlighting the imaging differences between choroid plexus carcinoma from papilloma.

\section{ABBREVIATION KEY}

$\mathrm{CSF}=$ cerebrospinal fluid

$\mathrm{WHO}=$ World Health Organization

$\mathrm{CPP}=$ choroid plexus papilloma

$\mathrm{aCPP}=$ atypical choroid plexus papilloma

$\mathrm{CPC}=$ choroid plexus carcinoma

TERT $=$ reverse-transcriptase protein subunit

INTRODUCTION

The choroid plexus is a richly vascularized secretory epithelium resulting from the invagination of the ependymal roof plate into the ventricular cavities by the blood vessels of the pia mater, producing the intraventricular cerebrospinal fluid (CSF) [1]. Tumors originating from choroid plexus are infrequent, representing less than $1 \%$ of all intracranial tumors [2]. According to the histological classification of the World Health Organization (WHO), they can be divided into choroid plexus papilloma (CPP), atypical choroid plexus papilloma $(\mathrm{aCPP})$, and choroid plexus carcinoma (CPC)[3]. The average annual incidence of all choroid plexus tumors 
is 0.3 out of 1,000,000 patients per year[4]. It is assumed that malignant tumors arise de novo [5]. Papillomas account for two thirds of choroid plexus tumors, they are generally benign and resection is usually curative. In contrast, carcinomas are malignant lesions with overall survival rates of 40 to $50 \%$ [6]

\section{CASE REPORT:}

A previously healthy 14 month old male presents with acute onset of seizures and a 10 days history of persistent vomiting and fever. Initial head computed tomography shows a heterogeneous intraventricular lobulated mass with punctate calcifications and focal intratumoral cystic areas, peripheral edema and associated hydrocephalus (Figure 1). An intraventricular shunt was placed for decompression. MRI showed a lobulated, intraventricular heterogeneous mass, with nodular enhancement and internal focal areas of diffusion restriction. The lateral posterior margin of the tumor extend though the ependymal wall into the periventricular parietooccipital white matter (Figure 2).

Patient had a partial tumor resection though right parietal approach, finding at surgery a lobulated mass with variable consistency (areas of friable and of solid tumor) and heterogeneous color going from pink to gray white. Due to its extension, poor definition of margins and friability behavior, total tumor resection is not possible.

Histopathology findings shows the presence of papillary structures with true fibrovascular stems, covered by a population of neoplastic cells, pleomorphic nuclei, with heavy stratification. Also, atypical "rhabdoid" cells are observed, with discrete cell borders, eccentric nuclei, and abundant eosinophilic cytoplasm Histopathologic diagnosis was choroid plexus carcinoma (Figure 3). He received intra-arterial neoadjuvant chemotherapy based on carboplatin, etoposide and ifosfamide, without initial adverse effects, waiting for a second surgical time to complete resection.

\section{DISCUSSION}

CPC are grade III brain tumors with poor prognosis. The oncogenesis of this lesion is unknown[7]. CPC could be confused with papillary ependymomas, malignant meningiomas and embryonal tumors in the pediatric age, occurring usually in children less than two years of age. In the adult, it can be confused with metastatic carcinoma. Almost all CPC occur in infants and children aged 2-4 years, with $70 \%$ initially diagnosed in children less than 24 months of age [2, 3,6]. Symptoms are usually due to hydrocephalus, which is of a lesser degree than that found in CPP $[1,8]$. The literature for pediatric populations indicate that 67 to $75 \%$ of all choroid plexus tumors are located in the lateral ventricles, $15 \%$ in the fourth ventricle, and $8 \%$ in the third ventricle; with no difference in location with respect to tumor histological classifications [9].

Imaging findings are not specific, showing an intraventricular lobulated mass, that may have punctate calcifications and homogeneous intense enhancement [5]. On CT scan CPCs appear iso-dense to hyper-dense, with calcification and avid enhancement. On MRI they usually appears iso or slight hyperintense on T1-weighted images, and are slightly hyperintense on T2-weighted images, showing typically intense enhancement. Serpentine signal flow voids may be present within or near the tumor, indicating enlarged blood vessels supplying or draining the tumor. Because papillomas are benign, they tend to expand the ventricle rather than invade adjacent brain, but both benign and malignant choroid neoplasms may show focal parenchymal invasion, signal characteristics and enhancement patterns that could make them indistinguishable [5]. Nevertheless, extensive parenchymal invasion with extension through the ependymal lining, and peritumoral vasogenic cerebral edema favor malignant lesions $[1,5]$. Both CPP and CPC presents elevated levels of Choline and absence of $\mathrm{N}$-acetyl aspartate by MR spectroscopy $[5,6]$.

The neuropathologic criteria established by the World Health Organization require: 1) obvious invasion of adjacent neural tissue with the infiltrating cells on a stromal base, with a diffuse and poorly defined pattern of growth; 2) loss of regular papillary architecture; and 3) evidence of cellular malignancy (increased mitotic activity, nuclear atypia, and necrosis) [10]. The epithelial sheath overlying the papillae can be redundant, exhibiting focal areas of stratification. Some nuclei can be hyperchromatic, and could find the presence of rhabdoid cells [5]. Immunohistochemistry is often inconclusive [5]. CPC could express 
carcinoembryonic antigen. Molecular findings include P53 mutations, Li-Fraumeni syndrome and mutations in reverse-transcriptase protein subunit (TERT) [9].

The surgical plan should include temporary or permanent resolution of hydrocephalus and definition of the tumor anatomic location, margins and blood supply. Tumors of the lateral ventricle or third ventricle are generally supplied by branches of the anterior or posterior choroidal arteries. Mass effect tends to displace the internal occipital artery and the basal vein of Rosenthal in an inferior direction[5]. Magnetic resonance angiography may provide more detailed images of the vascular supply of the tumor. One of the most important predictors of survival for CPC patients is initial total resection of the tumor, which can be difficult due to their invasive nature and also their softer and more friable behavior compared to papillomas $[3,5,6]$.

Although carcinomas rarely metastasize from the intracranial or intraspinal compartment, they can disseminate throughout the CSF pathways. Spinal MRI is an important modality for detecting spinal drop metastases. The overall incidence of metastases in CPC is 12-50\% [5]. Combined with adjunctive therapy, radiation, or chemotherapy, survival after surgery ranges from $67 \%$ to $91 \%[5,9]$.

\section{REFERENCES}

1. Guermazi, A., et al., Diagnostic Imaging of Choroid Plexus Disease: Pictorial Review. Clinical Radiology, 2000. 55(7): p. 503-516.

2. Tavallaii, A., et al., An extremely rare case of choroid plexus carcinoma in the third ventricle of an infant - Case report and review of the literature. Interdisciplinary Neurosurgery, 2020. 22 : p. 100873.

3. Zhou, W.J., et al., Clinical Features and Prognostic Risk Factors of Choroid Plexus Tumors in Children. Chin Med J (Engl), 2018.131 (24): p. 2938-2946.

4. Sun, M.Z., et al., Current management of choroid plexus carcinomas. Neurosurgical Review, 2014. 37 (2): p. $179-192$.

5. Gupta, N., Choroid plexus tumors in children. Neurosurgery Clinics of North America, 2003. 14 (4): p. 621-631.

6. Krieger, M.D., et al., Differentiation of choroid plexus tumors by advanced magnetic resonance spectroscopy. Neurosurg Focus, 2005.18 (6A): p. E4.

7. Carter, A.B., et al., Choroid plexus carcinoma presenting as an intraparenchymal mass. J Neurosurg, 2001. 95 (6): p. 1040-4.

8. Dung, L.T. and N.M. Duc, A Unique Case of Cerebellar Choroid Plexus Carcinoma. Can J Neurol Sci, 2021. 48 (4): p. 555-556.

9. Zaky, W. and J.L. Finlay, Pediatric choroid plexus carcinoma: Biologically and clinically in need of new perspectives. Pediatr Blood Cancer, 2018. 65 (7): p. e27031.

10. Shi, Y.Z., et al., Atypical choroid plexus papilloma: clinicopathological and neuroradiological features. Acta Radiol, 2017.58 (8): p. 983-990. 

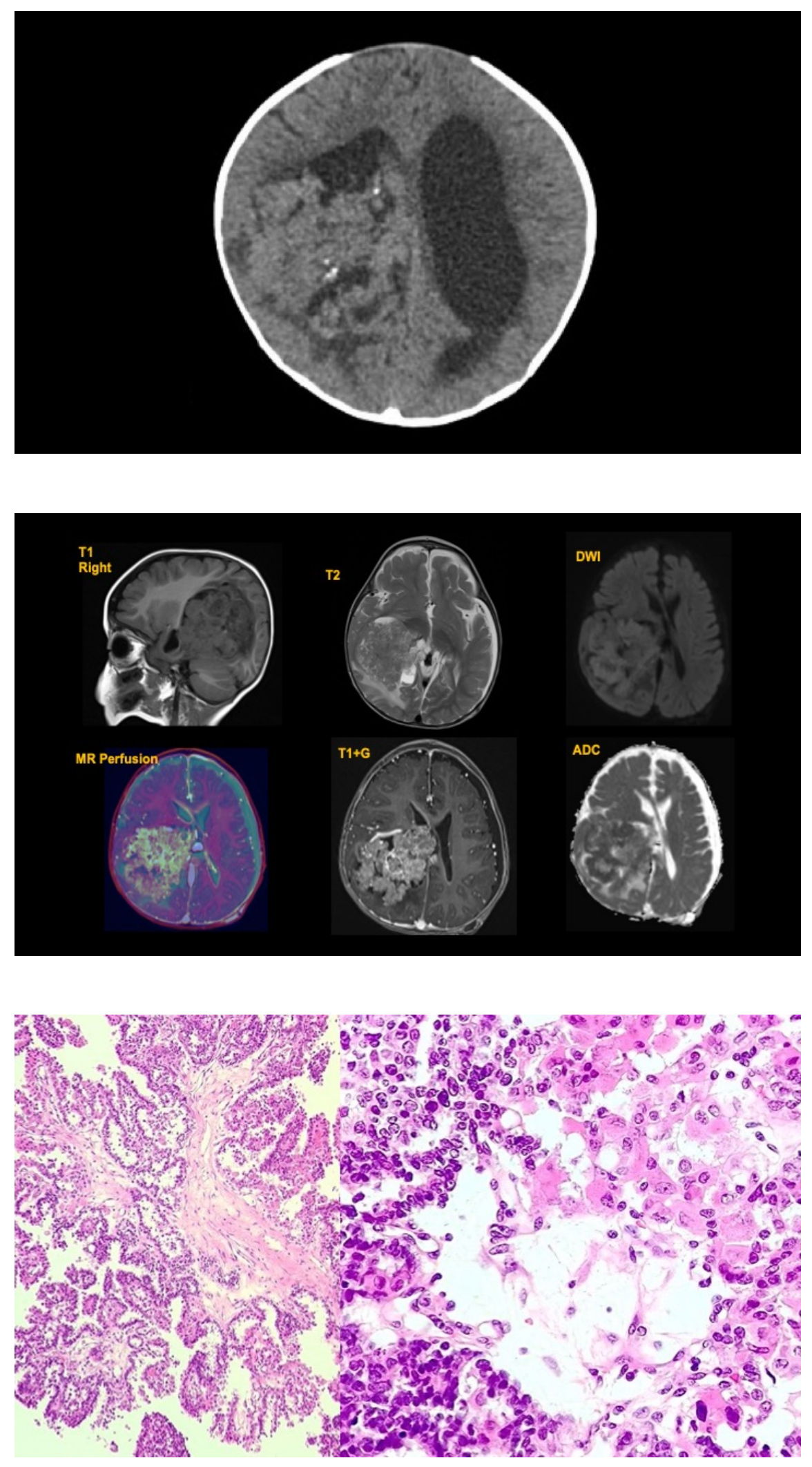